\title{
Observation of Four-Photon Orbital Angular Momentum Entanglement
}

\author{
B. C. Hiesmayr \\ Faculty of Physics, University of Vienna, Boltzmanngasse 5, A-1090 Vienna, Austria \\ M. J. A. de Dood and W. Löffler \\ Huygens-Kamerlingh Onnes Laboratory, Leiden University, P.O. Box 9504, 2300 RA Leiden, The Netherlands
}

(Received 23 September 2015; published 16 February 2016)

\begin{abstract}
We demonstrate genuine multipartite quantum entanglement of four photons in their orbital angular momentum degrees of freedom, where a high-dimensional discrete Hilbert space is attached to each photon. This can encode more quantum information compared to the qubit case, but it is a long-standing problem to entangle more than two such photons. In our experiment we use pulsed spontaneous parametric down-conversion to produce the photon quadruplets, which allows us to detect about one four-photon event per second. By means of quantum state reconstruction and a suitable witness operator we find that the photon quadruplets form a genuine multipartite entangled symmetric Dicke state. This opens a new tool for addressing foundational questions in quantum mechanics, and for exploration of novel high-dimensional multiparty quantum information applications such as secret sharing.
\end{abstract}

DOI: 10.1103/PhysRevLett.116.073601

Experimental control over systems where more than two particles are entangled is of interest for the study of foundational questions in quantum mechanics, and for multiparty quantum information schemes. So far, up to 14 particles have been entangled [1,2], but in each case the single-particle Hilbert space was strictly two-dimensional, i.e., qubits. For photons, the spatial degrees of freedom enable high-dimensional single particle spaces, which can be discretized in the photon orbital angular momentum (OAM). This enables implementation of novel quantum information protocols [3-5], and the study of fundamentally new quantum states [6,7]. To date, only two such multidimensional particles have been entangled [8,9] albeit with ever increasing dimensionality [10-12]; only in continuous variables, a first study goes beyond this [13]. Here, we use pulsed spontaneous parametric downconversion (SPDC) [14] to produce photon quadruplets that are entangled in their OAM, or transverse-mode, degrees of freedom $[8,15]$, and witness genuine multipartite Dicke-type entanglement [16-18]. Apart from addressing foundational questions [19-21], this could find applications in quantum metrology, imaging, and secret sharing [22,23].

Photons that are generated by near-collinear SPDC are correlated in several degrees of freedom and exhibit quantum entanglement. Apart from the well-known polarization degrees, the photons can also be correlated in their spatial degrees; this manifests itself in continuous wave vector or (the Fourier-related) position entanglement. This can be discretized using transverse optical modes, and a particular useful choice for experiments is the LaguerreGauss (LG) modes. Their azimuthal part factorizes and describes phase vortices [24] $\exp (i \ell \phi)$, where $\phi$ is the azimuth and $\ell=-\infty, \ldots, \infty$ determines the twisting number of the wave front, corresponding to an orbital angular momentum of $\ell \hbar$ per photon [25] (in addition to the spin angular momentum). The LG and the related Hermite-Gauss modes have well-known propagation dynamics; thus, they are suitable for the long-distance distribution of high-dimensional entanglement.

The SPDC interaction Hamiltonian matching is usually discussed in linear-momentum space [26], but can easily be rewritten in OAM space for a Gaussian pump beam and a rotational symmetric crystal on the wavelength scale (implying conservation of OAM [27] during the SPDC process):

$$
H=\sum_{\ell=-\infty}^{\infty} \frac{1}{2} i \kappa \hbar\left(a_{\ell}^{\dagger} a_{\bar{\ell}}^{\dagger}-a_{\ell} a_{\bar{\ell}}\right)
$$

where $a_{\ell}^{\dagger}$ is the creation operator for a photon with $\mathrm{OAM} \ell \hbar$, and $\bar{\ell} \equiv-\ell$ and $\kappa$ describes the strength of the nonlinear interaction. The first-order term of the series expansion of $|\Psi\rangle=\exp (-i t / \hbar H)|0\rangle$ produces a single pair of SPDC photons $\left|\Psi_{2}\right\rangle=\gamma \sum_{\ell=1}^{\infty}\left|1_{\ell} ; 1_{\bar{\ell}}\right\rangle$, which denotes two photons that are perfectly anticorrelated in their OAM [8] $\left(\left|n_{k}\right\rangle\right.$ is a state with $n$ photons in mode $\left.k\right)$. The single-pass amplitude gain $\gamma \propto \kappa t$ depends linearly on the pump beam intensity $I_{p}$. We explore here the next-order terms corresponding to the simultaneous production of two OAM photon pairs (for $\ell \neq 0$ modes):

$$
\left|\Psi_{4}\right\rangle \propto \gamma^{2}\left(\sum_{i, j=1, i \neq j}^{\infty}\left|1_{\ell_{i}} ; 1_{\ell_{j}} ; 1_{\bar{\ell}_{i}} ; 1_{\bar{\ell}_{j}}\right\rangle+2 \sum_{\ell=1}^{\infty}\left|2_{\ell} ; 2_{\bar{\ell}}\right\rangle\right) \text {. }
$$

This state can be seen as a result of interference in a double-pair emission process [28], which for production of multiple photon pairs is much more stable than 
interferometric SPDC [14] experiments. Because the fourphoton term given by Eq. (2) depends quadratically on the pump beam intensity, we use a focused picosecond pulsed pump laser with $\Delta t=2 \mathrm{ps}$ pulses. The group index mismatch of the pump and down-converted photons in our periodically poled potassium titanyl phosphate (PPKTP) crystal leads to a group velocity walk-off length $L_{g v}=1.3 \mathrm{~mm}$. To avoid the walk-off effect we use a $1 \mathrm{~mm}$ long crystal in the experiment. Furthermore, we use $1 \mathrm{~nm}$ wide bandpass filters for the down-converted photons to limit spectral (and temporal) labeling [29]. Focusing of the pump beam limits the number of spatial modes produced in SPDC, which can be characterized by the Schmidt number [30], so there is a trade-off between pump intensity and the number of produced transverse modes. We choose a pumpbeam waist of $50 \mu \mathrm{m}$ at the crystal, which results in $\sim$ nine down-converted transverse modes. We use for most measurements a pump beam power of $70 \mathrm{~mW}$, which corresponds to a peak intensity at the focus of $11.2 \mathrm{~kW} \mathrm{~cm}^{-2}$. This is close to the PPKTP damage threshold; above this, gray tracking was observed. Elevated temperatures or other materials, such as periodically poled lithium niobate, would enable the use of higher intensities. Since we use type-I SPDC, all produced photons have the same polarization. We use nonpolarizing beam splitters to separate them, and a combination of phase-only spatial light modulation and projection onto the core of single mode fibers to perform projective measurements in transverse-mode space [31,32], see Fig. 1 and the Supplemental Material [33].

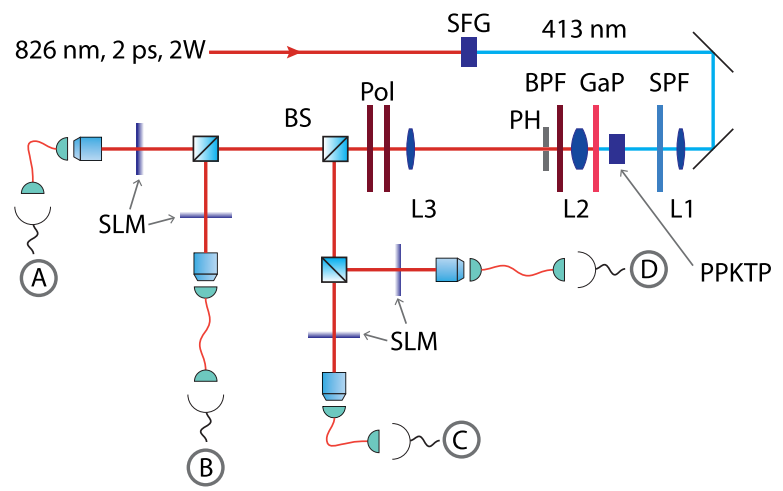

FIG. 1. Experimental implementation. A frequency-doubled (sum frequency generation, SFG) mode-locked picosecond laser is short-pass filtered (SPF) and focused (L1) into the PPKTP crystal. The SPDC photons are spectrally filtered with a GaP plate and a bandpass filter (BPF), and distributed with beam splitters (BSs) to the four equal detection units. The crystal facet is imaged with a telescope (L2 and L3) onto the spatial light modulators (SLMs), which in turn is far-field imaged onto the core of the detection single mode fibers connected to single photon counters. The pinhole $(\mathrm{PH})$ selects the 1st diffraction order of the SLM holograms. We explore the four-photon transverse-mode space by changing the holograms on the SLMs and recording fourfold coincidence events with a multichannel time tagging computer card.
To study the SPDC produced state from Eq. (2), we record four-photon correlations for different detection modes, pump powers, and relative detection times, shown in Fig. 2. We restrict ourselves to the fundamental Gaussian $(G)$ mode and the two-dimensional first-order mode space. The three mutually unbiased bases of the latter are $\left\{\mathrm{LG}_{+1}^{0}, \mathrm{LG}_{-1}^{0}\right\} \equiv\{R, L\}$ in the Laguerre-Gauss basis, $\left\{\mathrm{HG}_{0,1}, \mathrm{HG}_{1,0}\right\} \equiv\{H, V\}$ in the Hermite-Gauss basis, and $\left\{\mathrm{HG}_{0,1}^{45}, \mathrm{HG}_{1,0}^{45}\right\} \equiv\{D, A\} \quad$ in the $45^{\circ}$ rotated Hermite-Gauss basis, in analogy to the polarization case [34]. We have optimized the detection system for the firstorder modes; this leads to nearly equal single photon detection rates for the fundamental and first-order modes [Figs. 2(a) and 2(b)]. The fourfold coincidence rate $\Gamma$ for the case that all photons are produced by the same pump pulse [Fig. 2(c)] is highest if all four photons are projected onto the fundamental Gaussian mode $(|G G G G\rangle)$, and depends quadratically on the pump power as expected. We can now estimate the fraction of uncorrelated fourphoton detection events from comparing the case where all detectors "click" at the same time [Fig. 2(c)] to the case that two detectors detect photons from a different laser pulse [Fig. 2(d)], and we obtain $10 \%\left(\Gamma^{\Delta t=12.5 \mathrm{~ns}} / \Gamma^{\Delta t=0}\right)$. This is similar to the fraction of "forbidden" events (e.g., $\left.\Gamma_{H H H V} / \Gamma_{H H H H} \approx 0.1\right)$. We think the latter occur due to experimental imperfections; in contrast to polarization experiments we require here mode matching between all four detectors and the pump beam simultaneously. This argument is supported by the fact that both ratios are largely
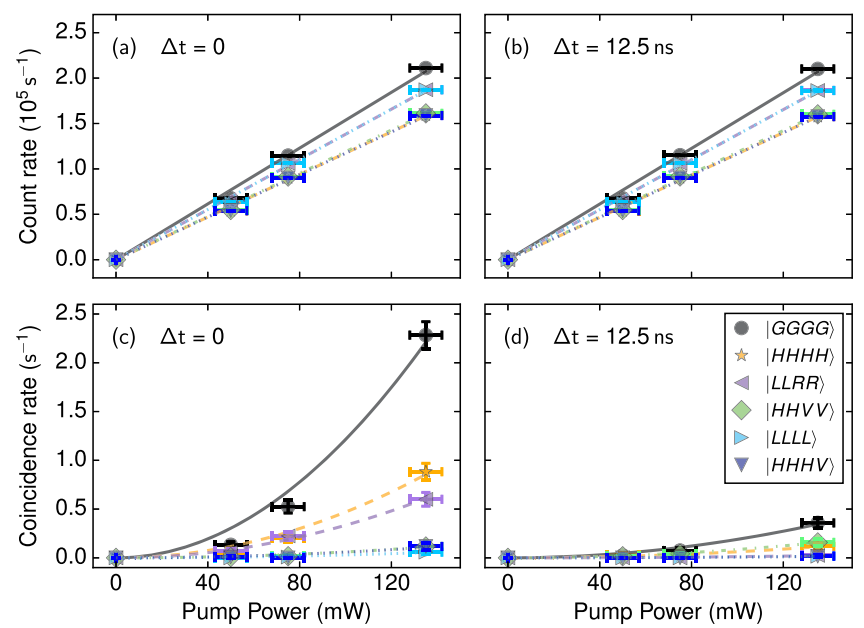

FIG. 2. Four-photon OAM state production. Pump-power dependent single-photon count rate (a),(b) and fourfold coincidence rate (c),(d), for various combinations of the detection transverse modes $\{G, H, V, L, R\}$. The single rates depend linearly on pump power, while the fourfold coincidence rates show quadratic dependency. The comparison between zero time delay (a),(c) and the case where photon 3 and 4 are delayed by 12.5 ns shows that most detected four-photon detection events are indeed due to four photons that were produced within a single pump pulse. 
independent of pump power, suggesting that contributions from higher-order multiphoton states are low.

These results support an intuitive explanation of the structure of Eq. (2), keeping in mind that the photons are produced in pairs: the first term in Eq. (2) that contains photons with different OAM $|\ell|$ occurs if the second pair is spontaneously emitted and uncorrelated to the first pair, while the second term corresponds to the case where the second pair is produced by a stimulated process giving a perfect clone of the first pair [35-38]. The different $|\ell|$ values in the first term in Eq. (2) is then simply a consequence of forbidden perfect quantum cloning; both terms together demonstrate the possibility of optimal quantum cloning in stimulated SPDC [39].

Now we study the quantum correlations of the SPDCproduced four-photon state, for which we focus on the twodimensional first-order mode space with $\operatorname{OAM} \ell= \pm 1$, and record fourfold coincidences for all detector mode combinations in each basis. Figure 3 compares experimental results and the theoretical prediction for the SPDCproduced state, which becomes in this case [Eq. (2)]

$$
\left|\Psi_{4}^{(2)}\right\rangle \propto\left|2_{\ell} ; 2_{\bar{\ell}}\right\rangle
$$

with $\ell=1$. Note that Eq. (3) is valid for any $\ell$, if we limit our detection to $\pm \ell$ modes (a 2D Hilbert space per photon). Experimentally, the beam splitters generate all possible permutations of photons (Fig. 1), so $\left|\Psi_{4}^{(2)}\right\rangle$ becomes in the detector basis

$$
\begin{aligned}
\left|D_{4}^{(2)}\right\rangle \propto & |\ell \ell \bar{\ell} \bar{\ell}\rangle+|\ell \bar{\ell} \ell \bar{\ell}\rangle+|\bar{\ell} \ell \ell \bar{\ell}\rangle+|\ell \bar{\ell} \bar{\ell} \ell\rangle \\
& +|\bar{\ell} \ell \bar{\ell} \ell\rangle+|\bar{\ell} \bar{\ell} \ell \ell\rangle,
\end{aligned}
$$

which is the symmetric Dicke state of $N=4$ photons with $N / 2=2$ excitations. This state is in particular interesting as it is robust to photon losses and has the largest distance from not genuine multipartite entangled states [40]. Entanglement in such states can easily be verified by measuring the total collective spin along the $x, y$ directions [40]; if $\left\langle\mathcal{W}_{4}^{(2)}\right\rangle=\left\langle J_{x}^{2}\right\rangle+\left\langle J_{y}^{2}\right\rangle>5$ [for $N=4, J_{i}=\frac{1}{2} \sum_{k} \sigma_{i}^{(k)}$, where, e.g., $\sigma_{y}^{(2)}=\mathbb{1} \otimes \sigma_{y} \otimes \mathbb{1} \otimes \mathbb{1}$, the state is nonseparable, i.e., entangled. We obtain experimentally $\left\langle\mathcal{W}_{4}^{(2)}\right\rangle=5.17 \pm 0.09$, thus verifying entanglement in the four-photon OAM state.

$\left\langle\mathcal{W}_{4}^{(2)}\right\rangle$ can also be used to detect genuine multipartite entanglement [16,17,41], if it violates $\left\langle\mathcal{W}_{4}^{(2)}\right\rangle \leq 7 / 2+$ $\sqrt{3} \approx 5.23$. The proposed theoretical state in Eq. (3), for which $\left\langle\mathcal{W}_{4}^{(2)}\right\rangle=6$, is indeed genuine multipartite entangled, but our experimental result does not violate this bound. We argue that experimental details are responsible: apart from spectral-filtering issues, we have here the extreme requirement that all four detectors have to be mode matched simultaneously. In contrast to experiments on polarization entanglement, here even small misalignment not only reduces count rates but also alters the measurement projectors by inducing small rotations in the respective single-particle Hilbert space, and the fourfold mode matching exponentially amplifies any misalignment. The experimentally obtained numerical values should therefore be seen as a lower limit only. We can correct for this partially if we have access to the full density matrix, as we now show.

We use tomographic quantum state reconstruction [42] to obtain the most likely density matrix $\rho$ describing the detected state. The experimental integration time is $120 \mathrm{~s}$ for each detector setting, and we additionally combine the data with the more accurate measurements of the basis-state correlations shown in Fig. 3. In Fig. 4 we compare the resulting experimental density matrix to the theoretical expectation (4); the fidelity is 0.62 . As a multipartite entanglement witness we use $I_{m}^{n}[\rho]$ proposed in Ref. [43] that is optimized for an $n$ particle Dicke state with $m$ excitations; it is also more resilient against noise compared to the collective-spin witness above. Violation of the inequality $I_{2}^{4}[\rho] \leq 0$ in our case indicates genuine multipartite entanglement of $\rho$; in our case we expect $I_{2}^{4}\left[\left|D_{4}^{(2)}\right\rangle\left\langle D_{4}^{(2)}\right|\right]=1$. See the Supplemental Material for an explicit form of the witness [33].
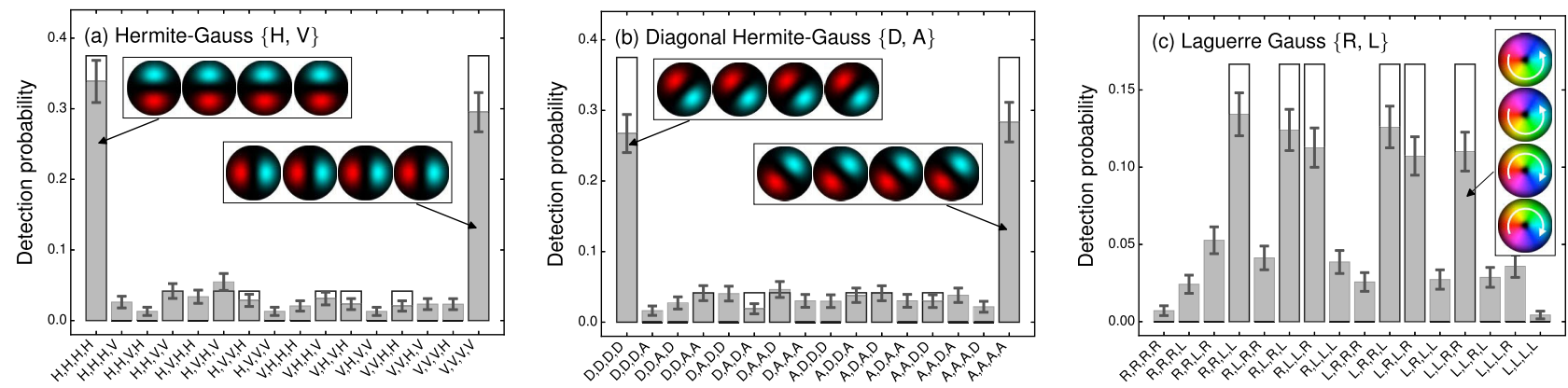

FIG. 3. Four-photon OAM correlations. Four-photon joint detection probabilities in each of the three mutually unbiased bases: (a) Hermite Gauss $\{H, V\}$, (b) diagonal Hermite Gauss $\{D, A\}$, and (c) Laguerre Gauss $\{R, L\}$. Labels indicate the phase and amplitude structure of the detected spatial modes. Gray bars: experimental data; the error bars show statistical errors. Boxes: theory. The experimental integration time per data point was 24 min. The probabilities are normalized to unity within each basis. 
(a)

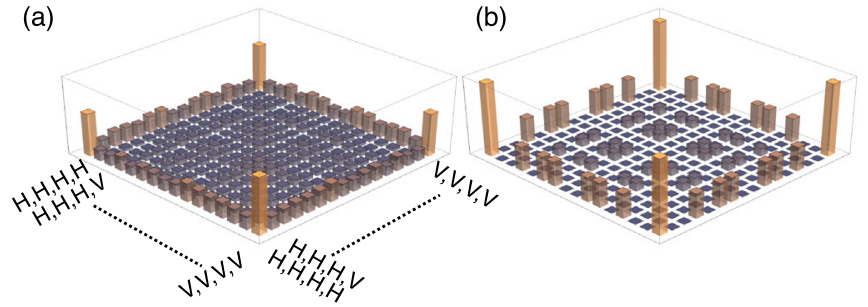

FIG. 4. The reconstructed OAM quantum state. Modulus of the reconstructed experimental (a) and theoretical (b) density matrix (vertical axis scale $[0,0.3]$ ). The discrepancy between experiment and theory is mainly caused by residual misalignment of the transverse-mode detectors.

For the analysis of the experimental density matrix, we apply local single-qubit rotations to search for maximum violation of the witness: such transformations are allowed to be applied to each part of a multipartite state because such local operations cannot change its entanglement properties. In addition, most entanglement witnesses, including $I_{m}^{n}[\rho]$ from Ref. [43], only detect entanglement optimally in a particular basis, and optimization over basis rotations has to be done. This can in principle also correct for experimental misalignments; however, we would need to have access to the full density matrix involving all spatial modes. Here, we can therefore only correct for relative rotations of the detectors. We optimize $I_{m}^{n}[\rho]$ by transformation of the experimentally determined (or theoretical) density matrix $\rho$ as follows: $\rho \rightarrow U \rho U^{\dagger}$, where $U=U_{1} \otimes$ $U_{2} \otimes U_{3} \otimes U_{4}$ with the generic unitary

$$
U_{i}=\left[\begin{array}{cc}
\exp \left(i \alpha_{i}\right) \cos \gamma_{i} & \exp \left(i \beta_{i}\right) \sin \gamma_{i} \\
-\exp \left[i\left(\alpha_{i}-\delta_{i}\right) \sin \gamma_{i}\right] & \exp \left[i\left(\beta_{i}-\delta_{i}\right) \cos \gamma_{i}\right]
\end{array}\right]
$$

for the real parameters $\left\{\alpha_{i}, \beta_{i}, \delta_{i}, \gamma_{i}\right\}$. These 16 parameters can simultaneously be optimized using robust unconstrained numerical optimization routines.

For three independently measured data sets, we obtain $I_{2}^{4}[\rho]=\{0.28,0.32,0.34\}$, thus giving strong indication of genuine four-photon OAM entanglement.

We have studied here the zero- and first-order modes with $\ell=\{0, \pm 1\}$; four-photon quantum correlations in this three-dimensional transverse-mode space are shown in the Supplemental Material [33]. Exploration of the rich correlations in the full higher-dimensional multipartite state (2) will require higher pump beam intensities and therefore the use of nonlinear crystals with a higher damage threshold, both within reach today. The four-photon OAM entangled state that we have produced and characterized here might open new possibilities and protocols in multiparty quantum secret sharing [22] with Dicke states in the sense that here more information per photon quadruplet can be exchanged or the security increased by the high-dimensional nature of the OAM or transverse-mode degrees of freedom [23]. Further, the spatial correlations carried by our multiphoton states might enable new options in quantum metrology, microscopy, and imaging.

We thank Martin van Exter for invaluable discussions. M. J. A. D. and W. L. acknowledge support from NWO and FOM. B. C. H. acknowledges support from the Austrian Science Fund (FWF 23627).

Note added.-Recently, after submission of our manuscript, a related study on three-photon OAM entanglement appeared [44].

*loeffler@physics.leidenuniv.nl

[1] T. Monz, P. Schindler, J. T. Barreiro, M. Chwalla, D. Nigg, W. A. Coish, M. Harlander, W. Hansel, M. Hennrich, and R. Blatt, Phys. Rev. Lett. 106, 130506 (2011).

[2] X.-C. Yao, T.-X. Wang, P. Xu, H. Lu, G.-S. Pan, X.-H. Bao, C.-Z. Peng, C.-Y. Lu, Y. A. Chen, and J.-W. Pan, Nat. Photonics 6, 225 (2012).

[3] S. P. Walborn, D. S. Lemelle, M. P. Almeida, and P. H. Souto Ribeiro, Phys. Rev. Lett. 96, 090501 (2006).

[4] B. P. Lanyon, M. Barbieri, M. P. Almeida, T. Jennewein, T. C. Ralph, K. J. Resch, G. J. Pryde, J. L. O'Brien, A. Gilchrist, and A. G. White, Nat. Phys. 5, 134 (2009).

[5] H. Bechmann-Pasquinucci and W. Tittel, Phys. Rev. A 61, 062308 (2000).

[6] B. C. Hiesmayr and W. Löffler, New J. Phys. 15, 083036 (2013).

[7] W. Wieczorek, C. Schmid, N. Kiesel, R. Pohlner, O. Guhne, and H. Weinfurter, Phys. Rev. Lett. 101, 010503 (2008).

[8] A. Mair, A. Vaziri, G. Weihs, and A. Zeilinger, Nature (London) 412, 313 (2001).

[9] A. Vaziri, G. Weihs, and A. Zeilinger, Phys. Rev. Lett. 89, 240401 (2002).

[10] A. C. Dada, J. Leach, G. S. Buller, M. J. Padgett, and E. Andersson, Nat. Phys. 7, 677 (2011).

[11] M. Krenn, M. Huber, R. Fickler, R. Lapkiewicz, S. Ramelow, and A. Zeilinger, Proc. Natl. Acad. Sci. U.S.A. 111, 6243 (2014).

[12] V. D. Salakhutdinov, E. R. Eliel, and W. Löffler, Phys. Rev. Lett. 108, 173604 (2012).

[13] L. K. Shalm, D. R. Hamel, Z. Yan, C. Simon, K. J. Resch, and T. Jennewein, Nat. Phys. 9, 19 (2013).

[14] A. Lamas-Linares, J. C. Howell, and D. Bouwmeester, Nature (London) 412, 887 (2001).

[15] J. C. Howell, R. S. Bennink, S. J. Bentley, and R. W. Boyd, Phys. Rev. Lett. 92, 210403 (2004).

[16] O. Gühne and M. Seevinck, New J. Phys. 12, 053002 (2010).

[17] M. Huber, F. Mintert, A. Gabriel, and B. C. Hiesmayr, Phys. Rev. Lett. 104, 210501 (2010).

[18] N. Kiesel, C. Schmid, G. Tóth, E. Solano, and H. Weinfurter, Phys. Rev. Lett. 98, 063604 (2007).

[19] T. Fritz, A. B. Sainz, R. Augusiak, J. Bohr Brask, R. Chaves, A. Leverrier, and A. Acín, Nat. Commun. 4, 2263 (2013).

[20] S. Popescu, Nat. Phys. 10, 264 (2014). 
[21] J. Carolan et al., Nat. Photonics 8, 621 (2014).

[22] M. Hillery, V. Buźek, and A. Berthiaume, Phys. Rev. A 59, 1829 (1999).

[23] L.-C. Yu, F.-L Lin, and C.-Y. Huang, Phys. Rev. A 78, 012344 (2008).

[24] J. F. Nye and M. V. Berry, Proc. R. Soc. A 336, 165 (1974).

[25] L. Allen, M. W. Beijersbergen, R. J. C. Spreeuw, and J. P. Woerdman, Phys. Rev. A 45, 8185 (1992).

[26] C. K. Hong and L. Mandel, Phys. Rev. A 31, 2409 (1985).

[27] S. P. Walborn, A. N. de Oliveira, R. S. Thebaldi, and C. H. Monken, Phys. Rev. A 69, 023811 (2004).

[28] H. Weinfurter and M. Źukowski, Phys. Rev. A 64, 010102 (2001).

[29] S. C. Yorulmaz, M. P. van Exter, and M. J. A. de Dood, Opt. Express 22, 5913 (2014).

[30] C. K. Law and J. H. Eberly, Phys. Rev. Lett. 92, 127903 (2004).

[31] N. R. Heckenberg, R. McDuff, C. P. Smith, H. RubinszteinDunlop, and M. Wegener, J. Opt. Quant. Electron. 24, S951 (1992).

[32] V. Y. Bazhenov, M. S. Soskin, and M. V. Vasnetsov, J. Mod. Opt. 39, 985 (1992).
[33] See Supplemental Material at http://link.aps.org/ supplemental/10.1103/PhysRevLett.116.073601 for details on the experiment and the entanglement witness.

[34] M. J. Padgett and J. Courtial, Opt. Lett. 24, 430 (1999).

[35] Z. Y. Ou, Phys. Rev. A 72, 053814 (2005).

[36] A. J. H. van der Torren, S. C. Yorulmaz, J. J. Renema, M. P. van Exter, and M. J. A. de Dood, Phys. Rev. A 85, 043837 (2012).

[37] H. De Riedmatten, V. Scarani, I. Marcikic, A. Acín, W. Tittel, H. Zbinden, and N. Gisin, J. Mod. Opt. 51, 1637 (2004).

[38] M. Eibl, S. Gaertner, M. Bourennane, C. Kurtsiefer, M. Zukowski, and H. Weinfurter, Phys. Rev. Lett. 90, 200403 (2003).

[39] C. Simon, G. Weihs, and A. Zeilinger, Phys. Rev. Lett. 84, 2993 (2000).

[40] G. Tóth, J. Opt. Soc. Am. B 24, 275 (2007).

[41] M. Bourennane, M. Eibl, C. Kurtsiefer, S. Gaertner, H. Weinfurter, O. Gühne, P. Hyllus, D. Bruß, M. Lewenstein, and A. Sanpera, Phys. Rev. Lett. 92, 087902 (2004).

[42] D. F. V. James, P. G. Kwiat, W. J. Munro, and A. G. White, Phys. Rev. A 64, 052312 (2001).

[43] M. Huber, P. Erker, H. Schimpf, A. Gabriel, and B. Hiesmayr, Phys. Rev. A 83, 040301(R) (2011).

[44] M. Malik et al., arXiv:1509.02561. 